\title{
Radial Head and Neck Allograft for Comminute Irreparable Fracture- Dislocations of the Elbow
}

\author{
SALVATORE BISICCHIA, MD; COSIMO TUDISCO, MD
}

\section{abstract}

Fracture-dislocations of the elbow can be difficult to treat, with unsatisfactory results in some cases. In general, it is preferable to preserve the fractured radial head when possible, but some patients present a unique treatment challenge because of extremely comminuted fractures and bone loss. In these cases, the only options available are radial head prosthesis or allograft. The authors present a case of a 45-year-old man with a fracture-dislocation of the left elbow that was treated with an allograft of the radial head and neck because of extreme comminution of the fracture. There have been a few reports about osteochondral allograft transplantation of the radial head, and they all included traumatic or posttraumatic cases treated with a frozen allograft. To the best of the authors' knowledge, this is the first report on the use of osteochondral allograft in the acute setting for the treatment of a comminuted fracture of the radius involving the whole head and neck. The clinical results were satisfactory at the final follow-up, although mild degenerative changes were present, the screws were coming loose, and the radial head had a slight valgus deformity. Radial head allograft can be an option in selected cases of acute fractures with severe comminution and bone loss that are not amenable to a stable internal fixation; for the young and active patient, who is not the best candidate for radial head resection; or in cases in which radial head arthroplasty is not feasible because of severe bone loss. [Orthopedics. 201x; $x x(x): x x-x x$.]

$\mathrm{F}$ Tracture-dislocations of the elbow can be difficult to treat, with unsatisfactory results in some cases. The aim of the surgery should be a stable concentric reduction that allows early mobilization and prevents joint stiffness. ${ }^{1}$ Both bone and soft tissues contribute to the stability of the elbow. The coronoid and elbow ligaments are primary restraints, and although the radial head is a secondary constraint, it takes on a critical role when the structural integrity of the coronoid and/or ligaments has been compromised. ${ }^{2,3}$ In general, it is prefer- able to preserve the fractured radial head when possible, ${ }^{4}$ but some patients present a unique treatment challenge because of extremely comminuted fractures and bone loss. In these cases, the only options available are radial head prosthesis or allograft. So far, the use of allograft around the elbow has been limited. ${ }^{5-8}$

The authors present a case of a 45-year-old man with a fracture-dislocation of the left elbow that was treated with an allograft of the radial head and neck because of extreme comminution of the fracture.

\section{CASE REPORT}

A 45-year-old male manual laborer in good physical condition and body shape was involved in an assault after a motor vehicle accident. He reported an injury to his left elbow, which was forced into hy-

The authors are from the Department of Orthopaedic Surgery, University of Rome "Tor Vergata," Rome, Italy.

The authors have no relevant financial relationships to disclose.

Correspondence should be addressed to: Cosimo Tudisco, MD, Department of Orthopaedic Surgery, University of Rome "Tor Vergata," Viale Oxford 81,00133, Rome, Italy (cosimo.tudisco@ uniroma2.it).

Received: July 30, 2015; Accepted: August 15, 2016.

doi: 10.3928/01477447-20160901-01 

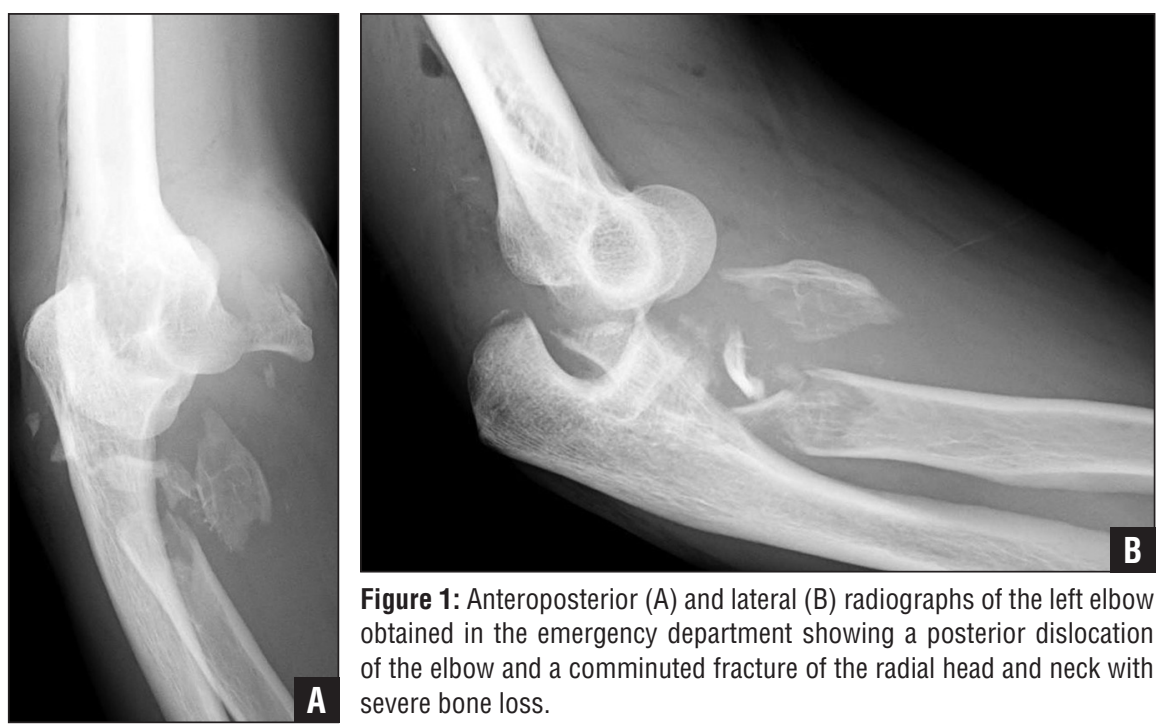

Figure 1: Anteroposterior (A) and lateral (B) radiographs of the left elbow obtained in the emergency department showing a posterior dislocation of the elbow and a comminuted fracture of the radial head and neck with severe bone loss.

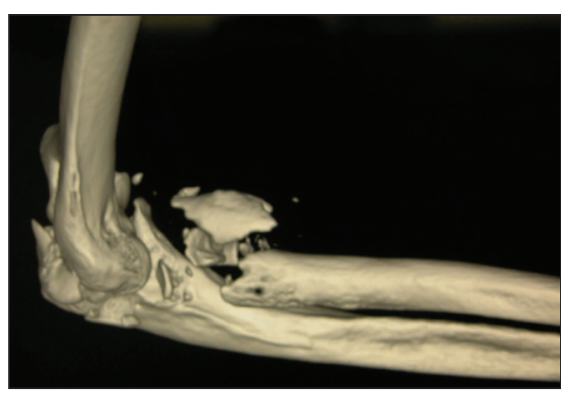

Figure 2: Preoperative computed tomography scan with 3-dimensional reconstructions further characterized the pattern of the fracture.

perextension against the opponent's knee. The patient immediately felt a sharp pain and a pop in his elbow. He was brought to the emergency department of the authors' institution, where radiographs were obtained showing an elbow dislocation with a comminuted fracture of the radial head and neck with severe bone loss (Figure 1). The elbow dislocation was reduced under sedation. The fracture was too comminuted for retention of the radial head by a stable internal fixation. Furthermore, bone loss of the radial neck was too severe for radial head arthroplasty. Radial head resection has been reported to provide good results in isolated fractures of young patients. ${ }^{9}$ In some of these cases, elbow instability may be observed following radial head resection and is usually secondary to

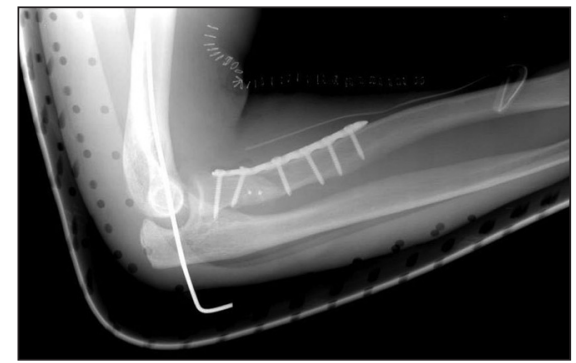

Figure 3: Postoperative lateral radiograph showing the elbow temporarily fixed with a transolecranon $\mathrm{K}$-wire and immobilized in a posterior long-arm splint.

unrecognized lateral ulnar collateral ligament deficiency. ${ }^{10}$

For these reasons, a radial head osteochondral allograft was recommended. During the preoperative workup, radiographs of the left wrist were obtained to rule out Essex-Lopresti injury. A computed tomography scan with 3-dimensional reconstructions was obtained for the injured side to better characterize the pattern of the fracture (Figure 2). Radiographs were also obtained for the contralateral side for allograft sizing, as per tissue bank protocol.

With the patient under general endotracheal anesthesia, an anterolateral approach to the left elbow according to Henry was performed 6 days after the injury. With the forearm in full supination, the posterior interosseous nerve was isolated from proximal to distal along its course on the arcade of Frohse. This nerve was retraced and protected during surgery. An avulsion of the distal biceps tendon was observed during surgery, and the radial tuberosity was fractured. The distal biceps brachii tendon was loaded with 2-0 Vicryl (Ethicon Inc, Somerville, New Jersey) sutures for subsequent reinsertion. The lateral collateral ligament appeared torn and frayed. The capsule was extensively damaged and disrupted because of the trauma. All fragments of the fracture were removed and put together with $\mathrm{K}$ wires on the back table to reconstruct the proximal radius as much as possible. This was important to determine how much of a graft was necessary to restore radial length, and to confirm sizing of the allograft. At that point, a frozen whole radial bone allograft, previously thawed at room temperature, was secured on a jig, cut to the length needed (determined by preoperative planning and intraoperative findings), and fixed to the radial bone of the patient with a $3.5-\mathrm{mm}$ Limited Contact Dynamic Compression Plate (Synthes, West Chester, Pennsylvania). The lateral collateral ligament was sutured to the graft using one 1.4-mm JuggerKnot anchor (Biomet Sports Medicine, LLC, Warsaw, Indiana); the annular ligament was impossible to repair. The distal tendon of the biceps brachii was reattached to the allograft with 2 suture anchors (Mini QuickAnchor Plus; DePuy Mitek, Raynham, Massachusetts). The elbow was temporarily fixed with a transolecranon 2-mm K-wire. A drain was placed, the wound was closed in the standard fashion, and the elbow was immobilized in a posterior long-arm splint (Figure 3).

The postoperative course was uneventful. The drain was removed on postoperative day 2 before discharging the patient from the hospital. He was seen in the clinic on postoperative day 14 for removal of the stitches and again at 6 weeks postoperatively for removal of the K-wire. Ra- 
diographs obtained at that time showed stable concentric reduction of the elbow and good alignment of the allograft with the shaft of the radius (Figure 4).

The patient was followed clinically and radiographically at 3 months, 6 months, 1 year, and then annually. At 6 weeks postoperatively, progressive range of motion in a hinged elbow brace was prescribed. Strengthening exercises with rubber bands and weights were allowed after 3 months. The patient achieved his definitive range of motion approximately 9 months postoperatively. At final follow-up, 4 years postoperatively, the patient showed good range of motion of his left elbow. He lacked approximately $20^{\circ}$ of supination, but this did not affect his work and/or everyday activities (Figure 5). His Mayo Elbow Performance Score was $85 / 100$. Radiographs at final followup showed stable concentric reduction of the elbow with mild degenerative changes. There was some osteolysis around the screws that were coming loose, and the radial head had partially collapsed into valgus (Figure 6). Mild screw loosening was noticed for the first time 3 years postoperatively, but subsequent evaluation at 4 years did not show progression.

At the final follow-up, the patient was completely asymptomatic and could perform heavy-duty activities. He was informed that in case of onset of symptoms related to the hardware itself, further imaging studies, laboratory tests, or surgical procedures may be considered. The mild valgus deformity of the elbow observed at the final follow-up was partially related to radial head collapse and partially related to the lack of extension (Figure 5).

\section{DISCUSSION}

To the best of the authors' knowledge, there have been few reports about osteochondral allograft transplantation of the radial head, and they all included traumatic or posttraumatic cases treated with a frozen allograft. ${ }^{5-7}$ Most of these authors
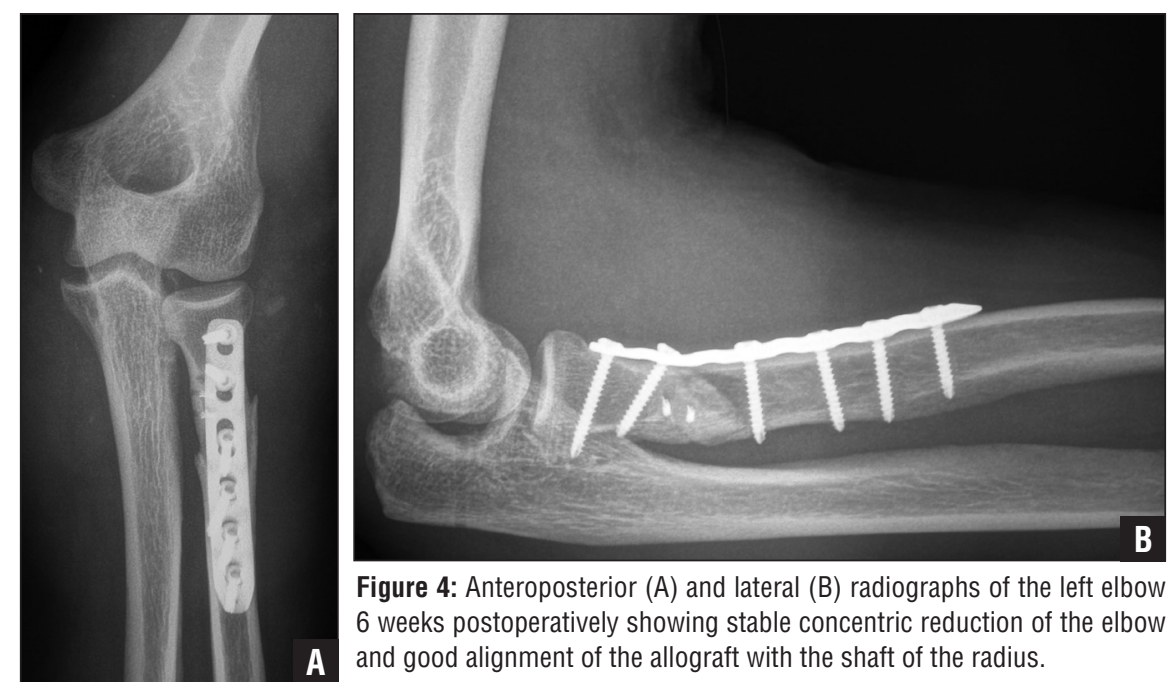

Figure 4: Anteroposterior $(A)$ and lateral $(B)$ radiographs of the left elbow 6 weeks postoperatively showing stable concentric reduction of the elbow and good alignment of the allograft with the shaft of the radius.
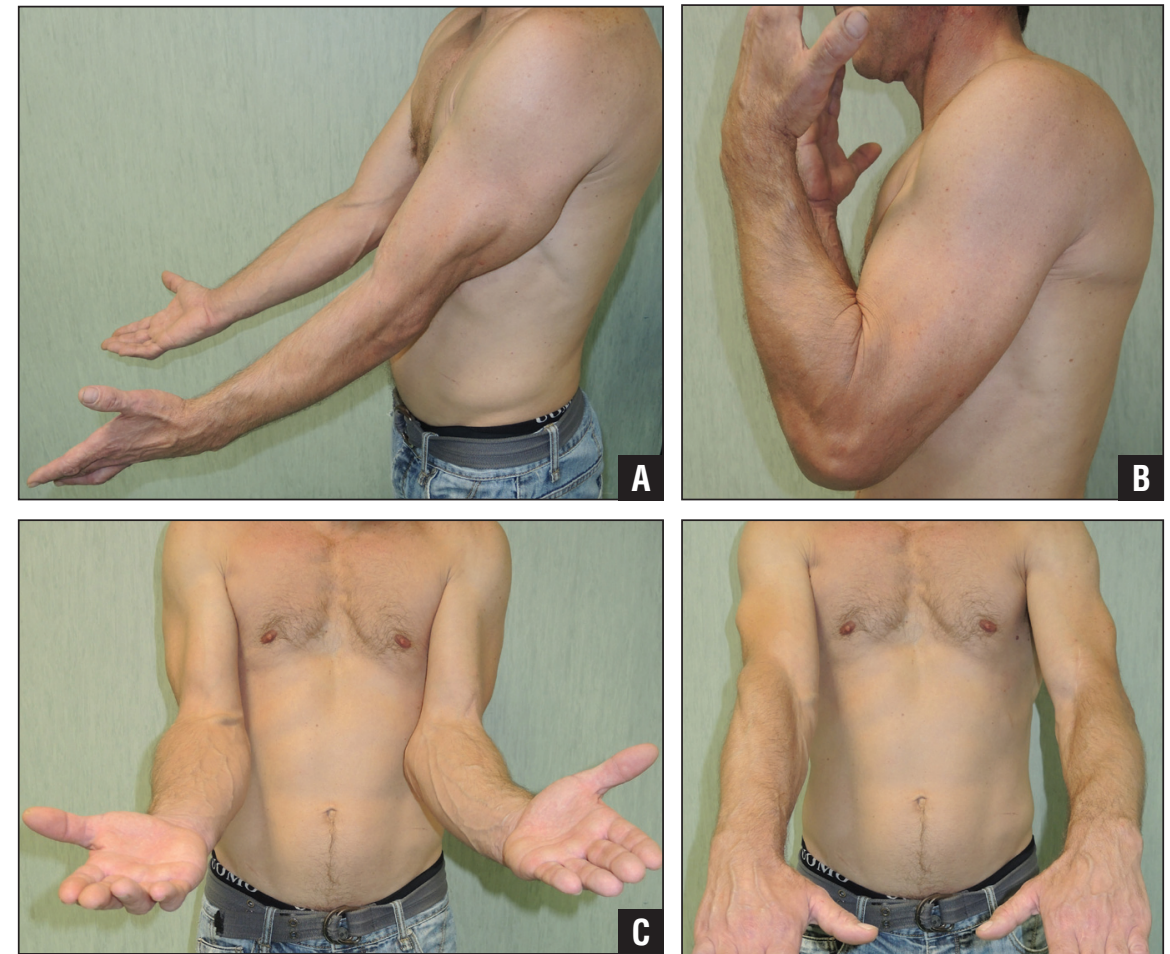

Figure 5: At final follow-up, 4 years postoperatively, the patient showed good range of motion of his left elbow in extension (A), flexion (B), and pronation (D). He lacked approximately $20^{\circ}$ of supination $(C)$, but this did not affect his work and/or everyday activities.

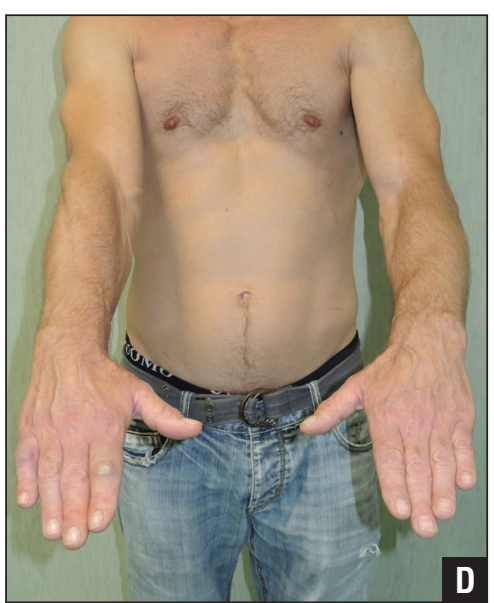

only reported their results for chronic injuries or revision cases. ${ }^{5,6}$

Szabo et $\mathrm{al}^{5}$ reported on 5 patients with symptoms related to proximal translation $(>1 \mathrm{~cm})$ of the radius following radial head excision (Essex-Lopresti le- sion). At a mean follow-up of 3 years (range, 1-7 years), all of the patients had relief of wrist and elbow pain and were satisfied with the outcome of the surgery. Both forearm and wrist range of motion had improved at follow-up. 

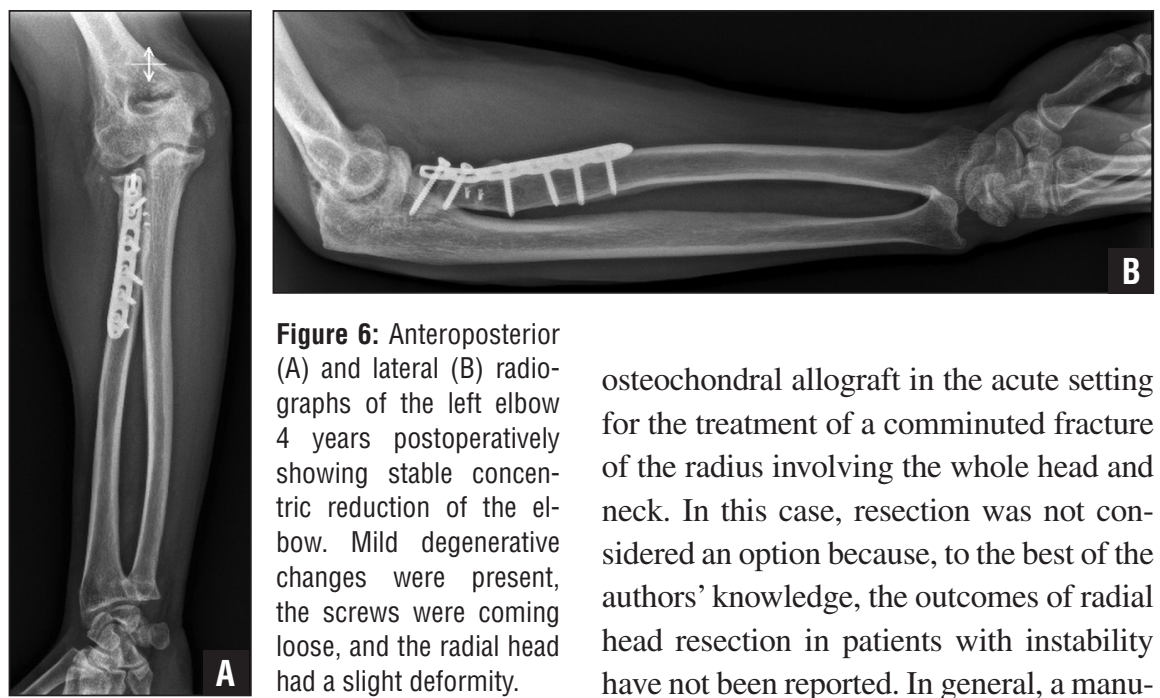

Figure 6: Anteroposterior (A) and lateral (B) radiographs of the left elbow 4 years postoperatively showing stable concentric reduction of the elbow. Mild degenerative changes were present, the screws were coming loose, and the radial head had a slight deformity.

Karlstad et $\mathrm{al}^{6}$ treated 4 patients (with 5 frozen allografts) affected by sequelae of Essex-Lopresti injuries. A lateral approach was used in all cases, with 3 of them requiring ulnar shortening. The results were not optimal, and the authors advocated the use of modular prosthetic radial head implants, which were not available when the patients were treated, or the creation of a 1-bone forearm, which was refused by all of the patients.

Turner et $\mathrm{al}^{7}$ reported for the first time on the use of allograft in the treatment of complex fracture-dislocations of the elbow, involving fractures of the coronoid and radial head, with collateral ligament disruption. They reviewed 8 patients treated with a partial radial head allograft (coronoid fracture and injury to collateral ligaments were also managed surgically). Only 2 of these patients were treated acutely at the authors' institution; the remaining 6 were chronic or revision cases referred to them 2 to 48 weeks after injury and after at least some treatment. Clinical and radiologic reviews confirmed union of the graft in all cases. None resorbed or collapsed. Three patients had resorption of the coronoid fragment resulting in poor outcomes, with 1 patient ultimately undergoing total elbow arthroplasty.

To the best of the authors' knowledge, this is the first case involving the use of osteochondral allograft in the acute setting for the treatment of a comminuted fracture of the radius involving the whole head and neck. In this case, resection was not considered an option because, to the best of the authors' knowledge, the outcomes of radial head resection in patients with instability have not been reported. In general, a manual laborer would benefit more from a reconstruction of the radial head; however, it was not possible in this specific case because of the severely comminuted fracture pattern and bone loss that prevented the surgeon from obtaining a stable internal fixation. With such a fracture pattern, resection may be an alternative for low-demand patients and for the elderly to decrease follow-up and potential complications such as infection or nonunion.

Radiographs at final follow-up showed stable concentric reduction of the elbow with mild degenerative changes. There was some osteolysis around the screws that were coming loose, and the radial head had partially collapsed into valgus. Radiographic evaluation did not show progression of screw loosening and valgus of the elbow between the third and the fourth years postoperatively. At the last followup, the patient was completely asymptomatic and could perform heavy-duty activities. The patient was continuing his follow-up visits to evaluate progression of screw loosening, angulation, and arthritis. In case of onset of symptoms related to the hardware itself, or in case of progression of these radiologic features, further imaging studies or laboratory tests may be considered (ie, to rule out infection and/ or nonunion). In particular, rescue procedures, such as radial head arthroplasty, radial head resection, or total elbow ar- throplasty, may be indicated depending on the patient's symptoms, imaging, and laboratory findings.

\section{CONCLUSION}

Radial head allograft can be an option in selected cases of acute radial head and neck fracture presenting with severe comminution and bone loss that are not amenable to a stable internal fixation; for the young and active patient, who is not the best candidate for radial head resection; or in cases in which radial head arthroplasty is not feasible because of severe bone loss.

\section{REFERENCES}

1. Morrey BF. Current concepts in the treatment of fractures of the radial head, the olecranon, and the coronoid. Instr Course Lect. 1995; 44:175-185.

2. Hotchkiss RN. Displaced fractures of the radial head: internal fixation or excision? $J \mathrm{Am}$ Acad Orthop Surg. 1997; 5(1):1-10.

3. O'Driscoll SW, Jupiter JB, King GJW, Hotchkiss RN, Morrey BF. The unstable elbow. J Bone Joint Surg Am. 2000; 82(5):724-738.

4. Papandrea RF, Morrey BF, O'Driscoll SW. Reconstruction for persistent instability of the elbow after coronoid fracture-dislocation. J Shoulder Elbow Surg. 2007; 16(1):68-77.

5. Szabo RM, Hotchkiss RN, Slater RR Jr. The use of frozen-allograft radial head replacement for treatment of established symptomatic proximal translation of the radius: preliminary experience in five cases. $J$ Hand Surg Am. 1997; 22(2):269-278.

6. Karlstad R, Morrey BF, Cooney WP. Failure of fresh-frozen radial head allografts in the treatment of Essex-Lopresti injury: a report of four cases. J Bone Joint Surg Am. 2005; 87(8):1828-1833

7. Turner RG, Rispoli D, Lopez-Gonzalez FM, O'Driscoll SW. Partial allograft replacement of the radial head in the management of complex fracture-dislocations of the elbow. $J$ Shoulder Elbow Surg. 2012; 21(3):396-404.

8. van Riet RP, Morrey BF, O'Driscoll SW. Use of osteochondral bone graft in coronoid fractures. J Shoulder Elbow Surg. 2005; 14(5):519-523.

9. Antuña SA, Sánchez-Márquez JM, Barco R. Long-term results of radial head resection following isolated radial head fractures in patients younger than forty years old. J Bone Joint Surg Am. 2010; 92(3):558-566.

10. Hall JA, McKee MD. Posterolateral rotatory instability of the elbow following radial head resection. J Bone Joint Surg Am. 2005; 87(7):1571-1579. 